\section{Discussion}

The method of holding the facemask on was simple and the mask could be quickly placed in position or removed. It was easy to change from mask CPAP to mask ventilation. The problems previously encountered with the fixation of masks ${ }^{*} 1_{15}$ seemed to have been avoided. No facial oedema necessitating repositioning of the mask or distortion of the head occurred, presumably because the pressure holding the mask against the face was evenly distributed around the head. Complications of the technique were minimal. Gastric distension was never severe, but we suspect that it might have been had higher lunginflating pressures been used. Undetected vomiting and aspiration is a theoretical risk, but so far as we are aware it never occurred. We suggest that this technique may have advantages over other methods of applying CPAP or mechanical ventilation to infants mildly affected by respiratory illnesses and that it should be useful in avoiding endotracheal intubation or reducing the length of time that infants with more serious illnesses have to be intubated.

We are grateful to the staff of the neonatal unit for their devoted care of the infants, and to $\mathrm{Mr} \mathrm{C} \mathrm{A} \mathrm{N} \mathrm{Evans} \mathrm{for} \mathrm{the} \mathrm{photograph.}$
Requests for reprints should be addressed to: Dr E O R Reynolds, Department of Paediatrics, University College Hospital, London WC1.

\section{References}

${ }^{1}$ Gregory, G A, et al, New England fournal of Medicine, 1971, 284, 1333.

2 Dunn, P M, Proceedings of the Royal Society of Medicine, 1974, 67, 245.

${ }^{3}$ Barrie, H, Lancet, 1972, 1, 776.

+ Kattwinkel, J, et al, Pediatrics, 1973, 52, 132

5 Caliumi-Pelegrini, G, et al, Archives of Disease in Childhood, 1974, 49, 228

${ }^{6}$ Kattwinkel, J, et al, Fournal of Pediatrics, 1975, 86, 588

Rhodes, P G, and Hall, R T, Pediatrics, 1973, 52, 1.

${ }^{8}$ Helmrath, T A, Hodson, W A, and Oliver, T K, fournal of Pediatrics, 1970, 76, 202.

9 Swyer, P R, et al, paper presented to INSERM, Nancy, Sept 1973.

10 Chernick, V, and Vidyasagar, D, Pediatrics, 1972, 49, 753.

1 Bancalari, E, Gerhardt, T, and Monkus, E, Pediatrics, 1973, 52, 128

${ }_{12}$ Stahlman, M T, et al, fournal of Pediatrics, 1970, 76, 174.

13 Stern, L, et al, Canadian Medical Association fournal, 1970, 102, 595.

14 Vert, P, Andre, M, and Sibout, M, Lancet, 1973, 2, 319.

15 Pape, K, Armstrong, D, and Fitzhardinge, P, Pediatric Research, 1975, 9, 383.

16 Shaw, J C L, Pediatric Clinics of North America, 1973, 20, 333.

\title{
Effects of "natural oestrogen" replacement therapy on menopausal symptoms and blood clotting
}

\author{
JEAN COOPE, JEAN M THOMSON, L POLLER
}

British Medical fournal, 1975, 4, 139-143

eliminated in individual cases by oestrogen. The results of blood clotting studies indicated that natural oestrogen administration raised the levels of the extrinsic clotting factors VII and $X$ and accelerated the prothrombin time. The findings were similar to those observed after three months' synthetic oestrogen administration with oral contraception.

Long-term studies and epidemiological surveys of the clinical incidence of thrombotic and other sequelae are needed before large-scale oestrogen replacement treatment can be recommended.

two groups and given either three months' hormon treatment followed by three months' placebo or vice versa.

An appreciable amelioration of all symptoms on placebo made it difficult to assess the genuine value of oestrogen treatment during the period of study. Both groups made a dramatic clinical improvement during the first three months. Nevertheless, the symptoms of the 15 women who received oestrogen first returned after the cross-over to placebo without any suggestion of a placebo response. In contrast, the other group who took placebo first did not deteriorate after changing to oestrogen.

The menopausal index and the karyopyknotic index were not reliable guides to the need for oestrogen treatment. Hot flushes, however, were proportionately reduced on oestrogen and they seemed to be more readily

Group Practice, Bollington, Cheshire

JEAN COOPE, $\mathrm{MB}, \mathrm{CHB}$, general practitioner

Department of Haematology, Withington Hospital, Manchester M20 8LR

JEAN M THOMSON, PH.D., senior scientific officer

L POLLER, MD, FRCPATH, consultant haematologis!

\section{Introduction}

So-called "natural" oestrogens have been widely used in the treatment of menopausal symptoms, and long-term treatment is recommended by many workers. ${ }^{12}$ It is, however, important to decide whether this treatment is both effective and safe. The use of the term "natural" for oestrogens prepared from equine urine is questionable but we have used the term because the product is customarily described in this way.

Many adverse effects occur in women taking synthetic oestrogens. We have shown that oral contraceptive preparations containing synthetic oestrogen accelerate blood clotting and platelet aggregation, ${ }^{3}{ }^{4}$ which may cause the increased risk of thromboembolism. It has been suggested that natural oestrogens may be less harmful than synthetic preparations because they have less effect on the electrophoretic mobility of platelets. ${ }^{5}$ Claims have also been made that natural oestrogens do not accelerate blood clotting but these require confirmation by laboratory studies.

Our double-blind cross-over study in women given natural oestrogens and placebo alternately was designed to discover whether menopausal symptoms respond to oestrogens. Objective assessment of changes in weight, blood pressure, blood clotting, and platelet aggregation were performed during the study. 


\section{Methods}

Women in a single general practice complaining of symptoms that were possibly menopausal were considered for inclusion in the study. They came from a mixed rural and industrial practice composed of 6500 patients and presented at the surgery with a variety of symptoms which were thought to be due to the menopause. Patients were seen several times, but only those who had complained of symptoms for over six months were admitted to the trial. Initial assessment was by the menopausal index. ${ }^{6}$ This scoring device allocates numbers in relation to the severity of symptoms and gives added weight to symptoms particularly associated with the menopause. Reduction of the score is believed to be a reliable guide to treatment. The following individual components of the menopausal index were also assessed separately: hot flushes (recorded weekly by the patient), paraesthesiae, insomnia, nervousness, depression, dizziness, weakness, arthralgia, headaches, palpitations, and formication.

Severity of symptoms was measured in all patients by one of us (JC) as follows: 3 = severe-incapacitating, causing inability to work or mix socially; $2=$ moderately severe-limiting social or other activities but able to work; $1=$ mildly unpleasant but not limiting activity; $0=$ no symptoms.

The severity of each symptom was multiplied by its "weighted" factor to provide a numerical conversion factor, the sum of these giving the menopausal index score. The weekly hot flush count was also recorded by the patient and was analysed separately.

We attempted an assessment of sexual function based on (a) local effects on the genital tract, $(b)$ the effect on libido, and (c) psychological effects on the marital relationship. The following clinical details were recorded: length and severity of symptoms, the date of the last menstrual period, previous history of thromboembolic disease, breast or genital cancer, and previous hysterectomy or oophorectomy. Patients with a history of thromboembolism or breast or genital cancer were excluded. Weight and blood pressure were recorded. Clinical examination included a screening for breast and genital cancer.

Five patients left before completion of the study. One patient moved and was lost to the trial, and two on oestrogen failed to attend for the clinical assessment at three months. Two patients with long psychiatric histories developed severe headaches and depression; the code was broken and they were found to be on oestrogen. They were consequently withdrawn from the trial.

Thirty women, aged 40 to 61 years (mean 52 years), remained in the study. There was no recognizable association between symptoms and social class, parity, or marital status. The women consisted of a mixed perimenopausal and postmenopausal group. Four were still menstruating regularly but five were only doing so at long irregular intervals. Ten had experienced a natural menopause between one and 10 years previously. Eleven patients had undergone hysterectomies and four had oophorectomies also.

The following baseline laboratory tests were performed on each patient; blood count, blood coagulation and platelet studies, blood group analysis, urine analysis, Ayre's smear, and vaginal cytology. All patients came to the haematology laboratory for their blood tests. Venepunctures were performed after the women had rested for at least 15 minutes. Blood coagulation and platelet studies were performed with a minimum of delay and always completed within one hour of collection of the blood sample.

The trial was conducted on a double-blind cross-over basis. Patients were randomly allocated to two groups each consisting of 15 patients. One group (group 1) received natural conjugated oestrogens (Premarin $1.25 \mathrm{mg} / \mathrm{d}$ ) during three 21 -day courses, with a break of seven days between each course. This group was then given placebo tablets containing lactose, with the same coating as Premarin, in the same dosage for a further three months. The other group (2) received placebo first and the natural conjugated oestrogens subsequently. A cyclic dosage scheme was used to prevent irregular bleeding, as withdrawal bleeding might be expected to occur in the week off treatment. The patients gave their informed consent, but neither they nor the medical and the ancillary staff were informed of the order of treatment.

\section{CLINICAL AND LABORATORY SUPERVISION}

Patients were seen monthly and their symptoms assessed. The weekly hot-flush count and the severity of all other symptoms were elicited separately. Patients were asked to report any side effects. Specific questions were asked at each visit about symptoms of pain or swelling in the legs. Weight and blood pressure were recorded every three months. A rise in diastolic blood pressure to over $90 \mathrm{~mm} \mathrm{Hg}$ or in systolic blood pressure to over $160 \mathrm{~mm} \mathrm{Hg}$ or an increase in weight of $3 \mathrm{~kg}$ were regarded as significant. At the end of the trial the patients were asked to give their subjective impression of their state of health during each three-month period.

Blood Clotting and Platelet Studies.-The following tests were performed before starting the trial and during the final week of both oestrogen and placebo administration, using the following techniques: ${ }^{7}$ prothrombin time using the national thromboplastin reagent (BCT); kaolin-activated partial thromboplastin (cephalin) time using a standardized reagent ${ }^{8}$ factor VII assay using a single lyophilized batch of beagle factor VII deficient substrate $;^{9}$ and factor $\mathrm{X}$ assays, using a factor $\mathrm{X}$ deficient substrate prepared in the laboratory that was matched against a severe human defect of factor $\mathrm{X}$. Because of the limited supplies it was not possible to use the same batch throughout. To compensate, therefore, for any possible interbatch variation each individual patient's factor $\mathrm{X}$ assay result was expressed as a percentage of a parallel control. Thrombelastography, reaction time ( $r)$, thrombin phase ( $k$ value), and maximum amplitude (ma value); platelet aggregation time (Chandler's tube technique), haemoglobin, and platelet count were also assessed each time.

Vaginal Cytology.-At the start of the trial vaginal smears were repeated every week for six weeks (except during menstruation) to indicate cyclic changes, and thereafter at the cross-over and completion of the trial. The proportion of oestrogenized superficial cells to intermediate and parabasal cells was determined and expressed as a percentage of the total cell count to give the karyopyknotic index (KI). Initial values were taken as the mean of the six pretreatment readings.

\section{Results}

\section{CLINICAL EVALUATION}

Menopausal index scores varied initially from 1 to 26 , the highest scores occurring in women with intact ovaries and uteri who had experienced a natural menopause from one to 10 years previously. The number of women severely affected is shown in table I. The individual symptom scores and the menopausal index score were reduced by the two treatment regimens during the first three months.

TABLE I-Severity of menopausal symptoms and clinical response to oestrogen and placebo during first three months of study

\begin{tabular}{|c|c|c|c|c|c|}
\hline Symptom & \begin{tabular}{|c|} 
No. \\
Moderately \\
or \\
Severely \\
Affected
\end{tabular} & $\begin{array}{l}\text { No. } \\
\text { Mildly } \\
\text { Affected }\end{array}$ & $\left|\begin{array}{c}\text { No. } \\
\text { Completely } \\
\text { Relieved } \\
\text { on } \\
\text { Placebo }\end{array}\right|$ & \begin{tabular}{|} 
No. \\
Completely \\
Relieved \\
on \\
Oestrogen
\end{tabular} & $\begin{array}{c}\text { P Value } \\
\text { Oestrogen }{ }^{\prime} \\
\text { Placebo }\end{array}$ \\
\hline 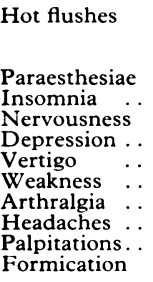 & $\begin{array}{r}6 \\
2 \\
14 \\
12 \\
4 \\
9 \\
8 \\
6 \\
5 \\
1\end{array}$ & $\begin{array}{r}14 \\
17 \\
8 \\
5 \\
6 \\
13 \\
9 \\
9 \\
11 \\
8\end{array}$ & $\begin{array}{l}4 \\
6 \\
4 \\
4 \\
4 \\
5 \\
3 \\
5 \\
3 \\
2\end{array}$ & $\begin{array}{r}6 \\
7 \\
10 \\
5 \\
3 \\
9 \\
5 \\
5 \\
9 \\
4\end{array}$ & $\begin{array}{c}0.78 \\
\text { (proportional } \\
\text { reduction }=0.04 \text { ) } \\
0.14 \\
0.25 \\
0.12 \\
0.93 \\
0.90 \\
0.11 \\
0.26 \\
0.16 \\
0.21 \\
0.30\end{array}$ \\
\hline $\begin{array}{l}\text { Menopausal } \\
\text { index score }\end{array}$ & 21 & 9 & 2 & 3 & 0.07 \\
\hline
\end{tabular}

The menopausal index score was greatly reduced by both placebo and oestrogen but the difference between these was not significant. There was, in fact, a significant improvement in the first three months in both groups (fig. 1), but those who received oestrogen first (group 1) did not show a placebo response in the second three months. In group 2, however, there seemed to be a further improvement when the patients changed to oestrogen but this was not significant. Individual patients in group 2, however, showed improvement in isolated symptoms such as paraesthesiae, arthralgia, palpitations, and depression during oestrogen administration.

Hot flushes varied initially from 0 to 140 per week in the 30 women. Nineteen women at the start of the trial were moderately or severely affected. Fig. 2 shows the great improvement in both groups during the first three months. Though there was no overall significant difference between the oestrogen and placebo response 10 women on 


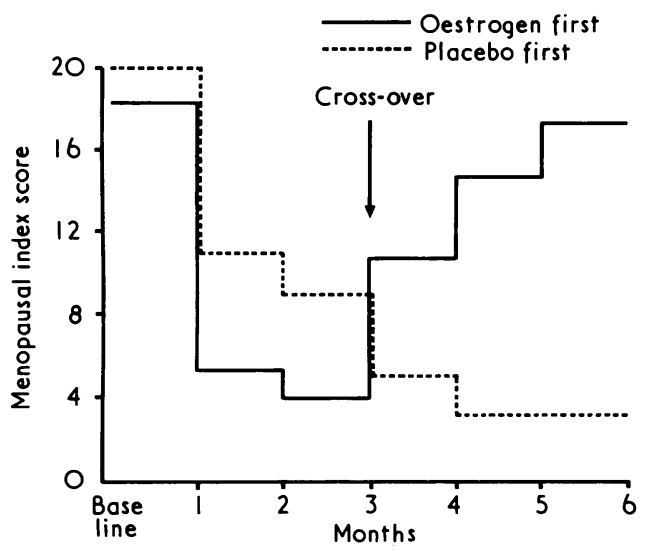

FIG. 1-Mean menopausal index scores groups 1 and 2.

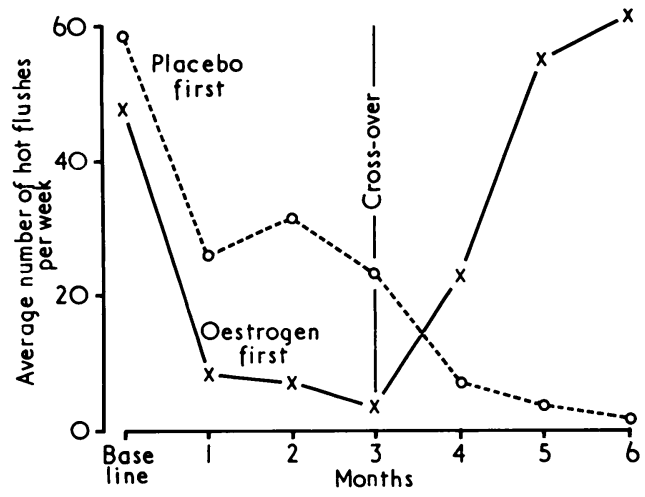

FIG. 2-Average number of hot flushes per week in groups 1 and 2 .

oestrogen compared with only four on placebo noticed total disappearance of hot flushes during the first three months. After the exclusion of three patients who had no hot flushes at the onset of the trial, however, the proportional reduction in hot flushes on oestrogen compared with placebo reached significance $(P=0.04)$.

In the second three months in group 1 hot flushes greatly increased when placebo was substituted for oestrogen $(P=0.005)$. In group 2 the apparent clinical improvement was not statistically significant compared with the previous placebo response. The results, however, were considerably better than the hot flush count in women given placebo in the second three months.

Sexual Function.-It was difficult to measure the effect of the oestrogen on sexual function owing to uncontrolled variables such as bereavement and husband's illness or unemployment. Six women who had suffered from dryness of the vagina and dyspareunia were greatly improved. Clitoral or urethral swelling occurred in some cases and may have contributed to the development of dysuria and urinary infection in two patients. At the end of three months on oestrogen libido was not increased in any of the women and was reduced in four patients. Nevertheless, 22 of the women said they felt a sense of well-being on the oestrogen that often resulted in improved marital relationships.

Side Effects.- These are listed in table II and were not significantly increased on oestrogen. No cases of deep venous thrombosis were detected during the study but four women developed superficial

TABLE II-Side effects during placebo and natural oestrogen administration. Results are numbers of patient:

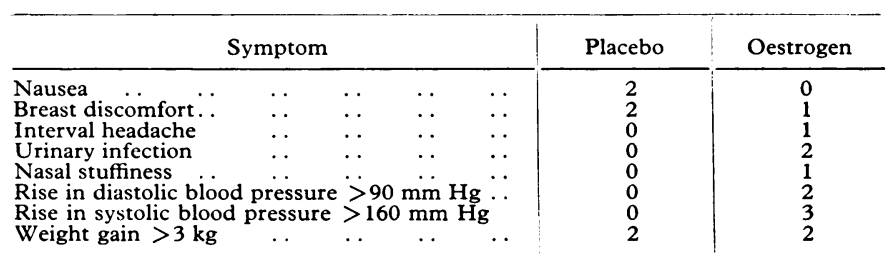

venous thromboses. One of these occurred in a group 2 patient during placebo administration. The other three occurred in women in group 1: one after 11 weeks on oestrogen and two within three weeks of changing to placebo. Withdrawal bleeding occurred during the cyclical gaps in all the perimenopausal patients and in two postmenopausal patients. It was not regarded as abnormal. Breakthrough bleeding did not occur in any of the patients during the trial. There was no significant increase in weight or blood pressure on oestrogens during the study.

\section{LABORATORY EVALUATION}

Prothrombin Time.-There was a significant difference between the baseline results and those in the treated group at three months and between those in the treated group and those in the placebo group after a similar time (figs. 3 and 4). There was, however, no significant difference between baseline prothrombin times and the values for the placebo group.

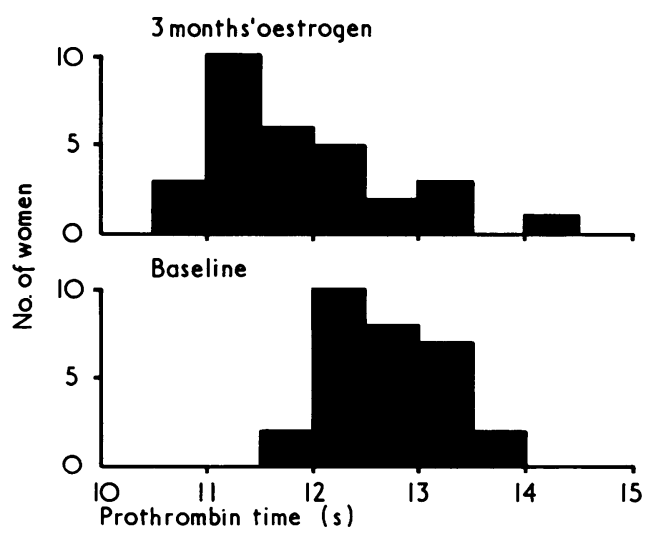

FIG. 3-Mean prothrombin times before (baseline) and after natural oestrogen administration.

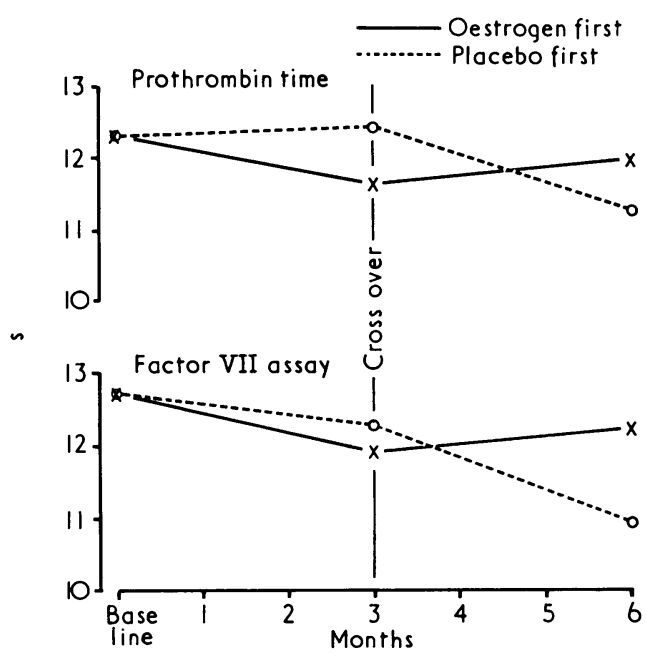

FIG. 4-Mean prothrombin times and factor VII levels in groups 1 and 2.

Partial Thromboplastin Time.-There was no significant change in either group during the study.

Factor VII Assay.-There was a significant difference between the baseline factor VII value and the results after three months on natural conjugated oestrogens. The placebo group also gave a result which was just significantly different, but when the group was subdivided (see fig. 4) into those who had received oestrogen first and those who had placebo first only the values in the former group were significantly increased-that is, the significant increase in factor VII was found only in the women who had received a three-month course of the oestrogen. 
Factor $X$ Assay.-The factor X values, expressed as a percentage of those of the parallel normal controls, showed a significant difference between the results in the oestrogen group and the baseline readings but no significant difference between those in the oestrogen group and those in the placebo group. Half of the latter group had, of course, received three months of oestrogen replacement before placebo.

Thrombelastography.-There was no significant change in either group in the three values measured during the study.

Platelet Aggregation Studies.-There was no significant difference between the baseline results and those in the oestrogen and placebo groups during the three-month administrations.

Haemoglobin and Platelet Count.-There was no significant alteration in these in either group.

Vaginal Cytology. - No significant association was found initially between the KI and the level of symptoms measured by the menopausal index or by the number of hot flushes. In both groups ( 1 and 2 ) the rise in $\mathrm{KI}$ when oestrogen was given was significantly greater than on placebo (fig. $5 ; \mathrm{P}<0.01$ ). There was a significant association between the $\mathrm{KI}$ and the menopausal index score only in group 1 when on oestrogen $(P<0.02)$. No significant association was found for group 2 on oestrogen or for either group on placebo. No significant correlation was found between changes in the $\mathrm{KI}$ and numbers of hot flushes.

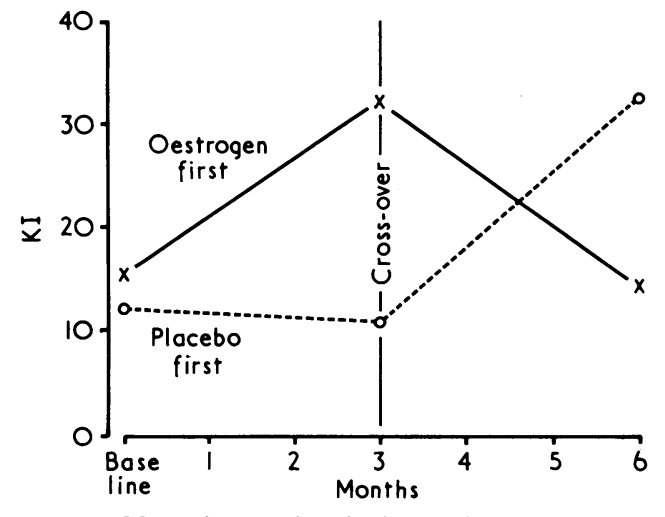

FIG. 5-Mean karyopyknotic index (KI) in groups 1 and 2.

\section{Discussion}

The definite placebo response of all symptoms in this doubleblind trial makes it difficult to assess the genuine pharmacological value of short-term oestrogen treatment. A dramatic clinical improvement occurred in both groups (1 and 2) during the first three months irrespective of whether they were given oestrogens or placebo. It was only after the cross-over period of the trial that any gross disparity between the oestrogen and placebo response was noted. The women who had received oestrogen first and then changed to placebo (group 1) deteriorated whereas the other group (2) did not. The key to this absence of placebo response in group 1 may be in the hot flush count. Having been told they would receive an inert preparation for three months and oestrogen for three months the relative failure of the placebo to eliminate hot flushes completely indicated to the women who had changed from oestrogen to placebo that they were taking the inert preparation. Although it was deemed inadvisable on ethical grounds, assessment might have been more objective if the patients had been told they were to be given two different types of hormone rather than oestrogen and an inert preparation. There might then have been some placebo effect in the second three months in group 1.

The menopausal index was not a useful guide to treatment as no significant difference was found between the reduction in the score produced by oestrogens and the reduction with placebo. Of the 11 symptoms included in the index only hot flushes showed a significant proportional but not absolute reduction on oestrogen compared with placebo after the exclusion of the three women who had no hot flushes at the start of the study.
This supports previous findings that only hot flushes were closely associated with the menopause, ${ }^{10}$ but the placebo response of the hot flushes confirms the findings of a recent report. ${ }^{11}$ It seems logical, therefore, to conclude that in spite of the advantageous effect of oestrogens in completely eliminating hot flushes in most patients the definite placebo response makes assessment of the genuine clinical value of oestrogen treatment of menopausal symptoms difficult to evaluate.

The KI level was not significantly related to the clinical state, and a rise did not reflect accurately an improvement in symptoms. It could not, therefore, be used as a reliable guide to the success of treatment.

The most unequivocal results of this study concern the effects of natural oestrogen on blood coagulation. It has been widely assumed, without adequate laboratory evidence, that unlike synthetic oestrogens, which raise levels of blood clotting factors, natural oestrogens have little or no effect on coagulation. Our findings are disturbing because after only threa months' treatment there was a pronounced acceleration of extrinsic clotting, as shown in the prothrombin time and factor VII assays. Factor $\mathrm{X}$, which is concerned in both extrinsic and intrinsic clotting, was also significantly increased but the changes were not as great as those in the extrinsic system assays. The adverse effects on clotting are substantially similar to those we have described after the same period of synthetic oestrogenprogestogen oral contraceptive administration. ${ }^{34}$ The results contrast unfavourably with the lack of acceleration of blood clotting by progestogen-only contraceptives. ${ }^{4} 12$

In the previous studies changes after administration of conventional oral contraceptives became progressively more marked after three months, as shown in a wider spectrum of tests, including assessments of the intrinsic clotting system and platelet aggregation. Further studies are in progress to determine whether the adverse effects of natural oestrogens on blood clotting show a similar progression.

The incidence of thromboembolism increases with age, and in menopausal women, who are substantially older than women on oral contraceptives, the acceleration of clotting during oestrogen treatment must cause concern. In these older women there is no compensatory reduction in mortality and morbidity from thromboembolism associated with pregnancy, such as obtained in younger women on oral contraceptives. There was a high incidence of superficial venous thrombosis in this study. Four cases occurred among the 30 women during the six months, though there was no obvious bias to either placebo or oestrogen groups. Thirty matched controls of a similar age group from the same practice over a similar period provided three cases. The numbers are, of course, too small to draw any conclusion on the danger of thrombosis from natural oestrogens and this can only be derived from larger long-term epidemiological studies, which seem to be urgently required.

This double-blind study monitored changes for only three months of equine oestrogen administration. Most of the patients were subsequently put on long-term oestrogen replacement and are being followed up. The incidence of irregular bleeding and thromboembolism in the long term requires adequate clinical assessment. It is necessary also to determine whether any more changes in blood clotting result from longer periods of oestrogen administration and whether changes in other values-for example, blood pressure, blood lipids, and bone density - can be detected in this group when compared with matched controls.

We thank Ayerst Laboratories Limited for the supplies of Premarin and placebo, Dr C Kingsley Bird, group pathologist, Macclesfield, Cheshire, Mr K Weston, chief technician, for the performance of the vaginal cytology, Mr A Gibbs, of the University of Manchester, for statistical help, and $\mathrm{Mr} S \mathrm{R}$ Armitage and technical staff at Withington. Professor V R Tindall provided helpful advice on the clinical protocol. L $\mathrm{P}$ was in receipt of a long-term programme grant from the Medical Research Council while the study was performed.

Request for reprint to be addressed to LP. 


\section{References}

${ }^{1}$ Wilson, R A, and Wilson, T A, fournal of the American Geriatrics Society, 1972, 20, 521 .

2 Gallagher, J C, and Nordin, B E C, Hormone Res?arch, 1973, 2, 98.

3 Thomson, J M, and Poller, L, British Medical fournal, 1965, 2, 270.

${ }^{4}$ Poller, L, Recent Advances in Thrombosis. Edinburgh, ChurchillLivingstone, 1973.

${ }^{5}$ Elkeles, R S, Hampton, J R, and Mitchell, J R A, Lancet, 1968, 2, 315.

6 Kupperman, H S, Blatt, M H G, and Wiesbaden, H, fournal of Clinical Endocrinology, 1953, 13, 88.
' Poller, L, Thomson, J M, and Thomas, $\mathrm{P}$ W, British Medical fournal, 1972, 4, 391.

8 Poller, L, and Thomson, J M, fournal of Clinical Pathology, 1972, 25, 1038.

9 Poller, L, Thomson, J M, and Sear, C H J, Fournal of Clinical Pathology, 1971, 24, 626.

${ }^{10}$ McKinlay, S M, and Jefferys, M, British Fournal of Preventive and Social Medicine, 1974, 28, 108.

11 Clayden, J R, Bell, J W, and Pollard, P, British Medical fournal, 1974, $1,409$.

12 Poller, L, et al, British Medical fournal, 1969, 1, 554.

\section{SHORT REPORTS}

\section{Ovarian ectopic pregnancy with IUCD}

We describe three cases of ovarian ectopic pregnancies with an intrauterine device (IUCD) in situ, and suggest that prostaglandins may have played a major part in this combination. The IUCD alters the ratio of the prostaglandins secreted, so that tubal peristalsis is reversed and the fertilised ovum is carried the opposite way.

\section{Case reports}

Three multigravidae presented with varying degrees of abdominal pains All of them had suffered from menstrual irregularity of recent duration, and all had had Lippes' loops inserted for over two years. $X$-ray films confirmed that the device was in position in each case. Laparotomy showed rightsided cystic ovarian swelling in one case and left-sided in the other two. The other ovary and both Fallopian tubes in each case were intact and uninvolved. Ovarian tissue was conserved in two cases, but the ovary had to be removed in one because of heavy, uncontrollable bleeding. The pathologist reported the histological features of pregnancy in the tissue excised.

\section{Discussion}

Ovarian pregnancy is very rare, a ratio of 0.7 ovarian per 100 ectopic pregnancies having been suggested. ${ }^{1}$ Women who wear an IUCD appear to have higher incidence, a ratio of one ovarian to nine ectopic pregnancies having been reported. ${ }^{2}$ Our cases fulfil the diagnostic criteria formulated by Spieglberg. ${ }^{3}$

Very little is known about the mode of action of the IUCD. It has been claimed that it distorts and distends the uterine cavity and thereby impairs its tonicity, or that it causes a foreign body reaction with leucocytic infiltration resulting in an environmental hostility to the ovum or that the macrophages phagocytoze the sperms. This spermatotoxic or blastotoxic hypothesis cannot explain the high relative frequencies of both tubal and ovarian ectopic pregnancies in patients fitted with IUCDs. Lehfeldt $e t$ al $^{1}$ suggested that the antifertility effect is maximal in the endometrium, weaker in the tubes, and absent beyond that - that is, about the ovary. This pattern, they thought, suggested direct enzymatic or chemical action, possibly by retrograde flow from the endometrium outwards.

We suggest that perhaps the chemical action is due to a substance resembling prostaglandin, which is secreted abundantly from the endometrium causing contractions and preventing implantation. Prostaglandin is also secreted from the fallopian tubes but in lesser amounts. Nevertheless, it is not secreted from the non-contractile ovaries.

Alternatively, possibly the IUCD alters the ratio of the amount of the prostaglandins secreted. Therefore, instead of the normal contraction of the proximal part of the tube and relaxation of the distal segments, causing a suction action which favours the entrance of the ovum from the abdominal cavity into the tube, and its retention in the oviduct until fertilisation, ${ }^{4}$ the reverse occurs and the reversed suction and peristaltic action of the tube (due to the effect of the altered ratio of prostaglandins) will carry the ovum the opposite way. This hypothesis could also explain the reported cases of peritoneal pregnancies in women fitted with IUCDs.

In most of the reported ovarian ectopic pregnancies in women with IUCDs, the right side has been affected. ${ }^{5}$ For tubal pregnancies it has been claimed that the right side is more often implicated possibly because of the proximity of the appendix. Such might also be the case in the ovarian ectopic pregnancy, but of our cases, two were left-sided and one right-sided; moreover, the free end of the Lippes' loop was found to be pointing towards the affected side in each case. Hence this might be an additional element in localising the condition.

${ }^{1}$ Lehfeldt, H, Tietze, C, and Gorstein, F, American fournal of Obstetrics and Gynecology, 1970, 108, 1005.

2 Tietze, C, American fournal of Obstetrics and Gynecology, 1966, 96, 1043.

${ }^{3}$ Novak, K R, and Woodruf, J D, Novak's Gynaecologic and Obstetric Pathology, p 446, 6th edn. Philadelphia, Saunders, 1967.

4 Sandberg, F, Ingelman-Sandberg, A, and Ryden, G, Acta Obstetrica et Gynecologica Scandinavica, 1965, 44, 585

5 Bozza, A T, and Horwitz, S T, American fournal of Obstetrics and Gynaecology, 1973, 117, 228.

St Catherine's Hospital, Birkenhead, Merseyside

D H DARWISH, MB, MRCOG registrar in obstetrics and gynaecology (now research fellow, Department of Obstetrics and Gynaecology, Liverpool University)

Liverpool Maternity Hospital and Liverpool Women's Hospital SAMIA T A SAAFAN, MB, CHB, senior house officer

\section{C shock: spontaneous reversion to sinus rhythm during anaesthesia with propanidid}

In the 1950s several derivatives of eugenol, the main constituent of oil of cloves and cinnamon leaf oil, were found to have short-acting anaesthetic properties. ${ }^{12}$ One of these, propanidid, causes sinus tachycardia and transient systemic hypotension, a decrease in stroke volume, and an increase in cardiac output as well as a slight fall in peripheral vascular resistance. ${ }^{34} \mathrm{We}$ have used propanidid as an intravenous anaesthetic agent in nine episodes of arrhythmia in seven patients requiring cardioversion. In four episodes in two patients (cases 1 and 2) the arrhythmia reverted to sinus rhythm after injection of propanidid.

\section{Case reports}

Case 1.-A 19-year-old Indian girl presented with a ventricular tachycardia $(200$ beats $/ \mathrm{min}$ ) and hypotension (B.P. $80 / 70 \mathrm{~mm} \mathrm{Hg}$ ). She reverted to sinus rhythm during induction of anaesthesia with propanidid, $400 \mathrm{mg}$ (see fig.). Two hours later the ventricular tachycardia recurred. It again reverted to sinus rhythm after a further $400 \mathrm{mg}$ propanidid. Subsequent epicardial mapping confirmed the presence of a re-entry ventricular tachycardia. A myocardial biopsy showed histological changes compatible with a giant cell myocarditis.

Case 2.-A 60 -year-old woman had a long history of paroxysmal supraventricular tachycardia. On previous occasions her arrhythmia had responded 